1 Selective adsorption and selective transport diffusion

\title{
2 of $\mathrm{CO}_{2}-\mathrm{CH}_{4}$ binary mixture in coal ultramicropores
}

$4 \quad{ }^{\mathrm{a}}$ School of Energy and Environmental Engineering, University of Science and Technology

$6 \quad{ }^{\mathrm{b}}$ State Key Laboratory of Multiphase Flow in Power Engineering, Xi'an Jiaotong University, Xi'an 710049, China

$8 \quad{ }^{\mathrm{c}}$ Beijing key laboratory of Energy Saving and Emission Reduction for Metallurgical Industry,

\section{* Corresponding author: \\ E-mail: yhfeng@me.ustb.edu.cn (Yanhui Feng)}

Tel: +86-010-62334971

Fax: +86-010-62329145 


\section{Supporting Information}

\section{Contents}

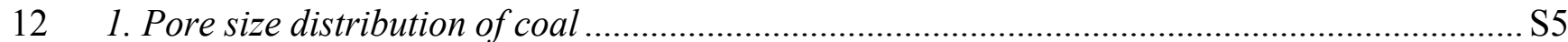

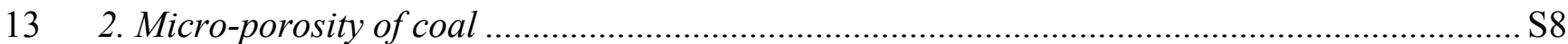

14 3. Formula of three distinct diffusion coefficients ............................................................... S9

15 4. Initial conformation for MD simulation .............................................................................. S12

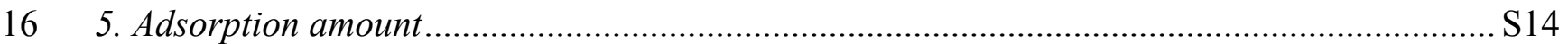

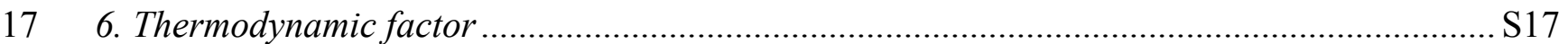

$18 \quad$ 7. Pressure dependence on diffusion coefficients .................................................................. S18 


\section{Supporting Information}

22 Table S1. Fraction of pore size distribution for every slice.......

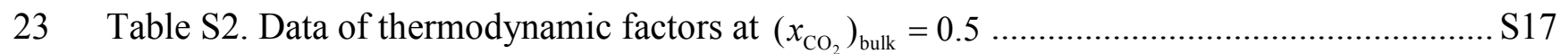

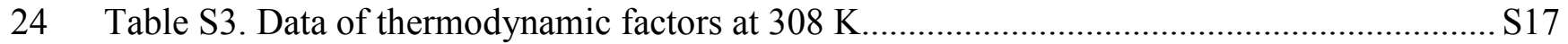

25

26 


\section{Supporting Information}

28 Figure S1. Simulation cell of coal with 30 slices. Slices AB1-AB10, BC1-BC10, and AC1-

$29 \mathrm{AC} 10$ are paralleled to $\mathrm{AB}$ plane, $\mathrm{BC}$ plane, and $\mathrm{AC}$ plane, respectively. In slices, the pores

30 are depicted in blue color

31 Figure S2. Pore size distribution of simulated coal model S7

32 Figure S3. Example of the molecular configuration sampled for diffusion of the $\mathrm{CO}_{2}-\mathrm{CH}_{4}$

33 mixture in the bituminous coal model. (a) Adsorption conformation obtained by GCMC

34 simulation. (b) Initial conformation for $\mathrm{MD}$ simulation. $\mathrm{CO}_{2}$ and $\mathrm{CH}_{4}$ molecules are

35 depicted in red and blue color, respectively.

36 Figure S4. Absolute adsorption isotherms of the $\mathrm{CO}_{2}-\mathrm{CH}_{4}$ mixture in coal ultramicropores

37 at $\left(x_{\mathrm{CO}_{2}}\right)_{\text {bulk }}=0.5$, i.e. $\left(x_{\mathrm{CH}_{4}}\right)_{\text {bulk }}=0.5$

38 Figure S5. Absolute adsorption isotherms of the $\mathrm{CO}_{2}-\mathrm{CH}_{4}$ mixture in coal ultramicropores

39 at $308 \mathrm{~K}$

40 Figure S6. Diffusion coefficients of the $\mathrm{CO}_{2}-\mathrm{CH}_{4}$ mixture in coal ultramicropores versus

41 Pressure at $298 \mathrm{~K}$ and $\left(x_{\mathrm{CO}_{2}}\right)_{\text {bulk }}=0.5$. The subscript ' 1 ' refers to $\mathrm{CO}_{2}$, and ' 2 ' refers to $\mathrm{CH}_{4}$.

42 The error bars were obtained from the slopes of the least squares fits S19 


\section{Supporting Information}

\section{Pore size distribution of coal}

46 To calculate the pore size distribution of coal, first, the simulation cell with 30 slices

47 paralleling to axis planes were selected and presented in Figure S1. Each slice was used to count

48 the pore sizes, and the results are list in Table S1. Then, the total pore size distribution of

49 simulated coal model could be considered as the average value of data among the 30 groups.

50 Finally, function of Gaussian distribution was used to fit the curve, the results are depicted in

51 Figure S2. The average pore diameter is $0.398 \pm 0.007 \mathrm{~nm}$ and in the scope of reported

52 microporous size of coal.

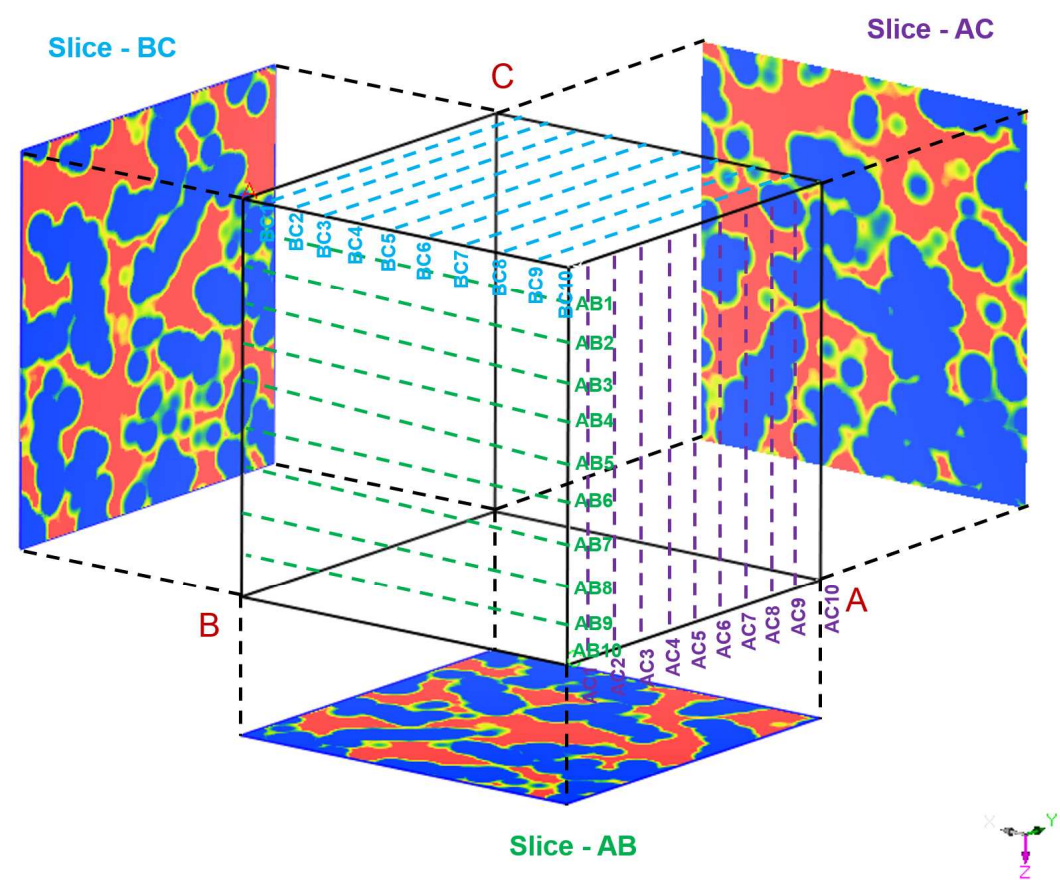

54 Figure S1. Simulation cell of coal with 30 slices. Slices AB1-AB10, BC1-BC10, and AC1-

$55 \mathrm{AC} 10$ are paralleled to $\mathrm{AB}$ plane, $\mathrm{BC}$ plane, and $\mathrm{AC}$ plane, respectively. In slices, the pores are

56 depicted in blue color. 


\section{Supporting Information}

Table S1 Fraction of pore size distribution for every slice.

\begin{tabular}{|c|c|c|c|c|c|c|c|c|c|c|}
\hline \multirow{2}{*}{$\begin{array}{l}\text { Diameter } \\
(\mathrm{nm})\end{array}$} & \multicolumn{10}{|c|}{ Fraction of pore size distribution (\%) } \\
\hline & $\mathrm{AB} 1$ & $\mathrm{AB} 2$ & $\mathrm{AB} 3$ & $\mathrm{AB} 4$ & AB5 & AB6 & $\mathrm{AB} 7$ & $\mathrm{AB} 8$ & AB9 & $\mathrm{AB} 10$ \\
\hline $0-0.1$ & 0.00 & 0.00 & 1.92 & 0.00 & 0.00 & 0.00 & 0.00 & 0.00 & 0.00 & 0.00 \\
\hline $0.1-0.2$ & 13.95 & 7.14 & 11.54 & 0.00 & 11.11 & 17.50 & 19.51 & 14.29 & 10.00 & 22.86 \\
\hline $0.2-0.3$ & 16.28 & 7.14 & 11.54 & 23.26 & 15.56 & 22.50 & 17.07 & 11.90 & 17.50 & 22.86 \\
\hline $0.3-0.4$ & 18.60 & 30.96 & 25.00 & 13.95 & 28.89 & 0.00 & 12.20 & 16.67 & 10.00 & 17.14 \\
\hline $0.4-0.5$ & 27.91 & 26.19 & 34.62 & 27.91 & 15.56 & 22.50 & 21.95 & 26.19 & 32.50 & 8.57 \\
\hline $0.5-0.6$ & 9.30 & 19.05 & 13.46 & 23.25 & 22.22 & 22.50 & 9.75 & 16.67 & 10.00 & 8.57 \\
\hline $0.6-0.7$ & 11.63 & 7.14 & 0.00 & 6.98 & 4.44 & 10.00 & 9.76 & 9.52 & 10.00 & 8.57 \\
\hline $0.7-0.8$ & 2.33 & 2.38 & 1.92 & 4.65 & 2.22 & 5.00 & 7.32 & 2.38 & 2.50 & 8.57 \\
\hline $0.8-0.9$ & 0.00 & 0.00 & 0.00 & 0.00 & 0.00 & 0.00 & 2.44 & 2.38 & 5.00 & 2.86 \\
\hline $0.9-1.0$ & 0.00 & 0.00 & 0.00 & 0.00 & 0.00 & 0.00 & 0.00 & 0.00 & 2.50 & 0.00 \\
\hline Diameter & $\mathrm{AC} 1$ & $\mathrm{AC} 2$ & $\mathrm{AC} 3$ & $\mathrm{AC} 4$ & AC5 & AC6 & $\mathrm{AC7}$ & $\mathrm{AC} 8$ & AC9 & $\mathrm{AC} 10$ \\
\hline $0-0.1$ & 0.00 & 13.95 & 0.00 & 0.00 & 0.00 & 0.00 & 0.00 & 0.00 & 0.00 & 0.00 \\
\hline $0.1-0.2$ & 23.91 & 34.88 & 5.00 & 16.28 & 8.82 & 16.22 & 9.76 & 9.09 & 12.50 & 25.00 \\
\hline $0.2-0.3$ & 13.04 & 23.26 & 20.00 & 16.28 & 2.94 & 18.92 & 14.63 & 15.91 & 12.50 & 20.83 \\
\hline $0.3-0.4$ & 21.74 & 23.25 & 12.50 & 18.60 & 17.65 & 21.62 & 24.39 & 22.73 & 15.00 & 18.75 \\
\hline $0.4-0.5$ & 17.39 & 2.33 & 20.00 & 23.25 & 11.76 & 13.51 & 17.07 & 22.72 & 17.50 & 12.50 \\
\hline $0.5-0.6$ & 10.87 & 2.33 & 12.50 & 9.30 & 32.36 & 5.41 & 14.63 & 18.18 & 12.50 & 10.42 \\
\hline $0.6-0.7$ & 8.70 & 0.00 & 7.50 & 11.63 & 17.65 & 8.11 & 9.76 & 6.82 & 20.00 & 6.25 \\
\hline $0.7-0.8$ & 4.35 & 0.00 & 17.50 & 2.33 & 5.88 & 10.81 & 4.88 & 4.55 & 2.50 & 4.17 \\
\hline $0.8-0.9$ & 0.00 & 0.00 & 0.00 & 2.33 & 2.94 & 2.70 & 4.88 & 0.00 & 7.50 & 2.08 \\
\hline $0.9-1.0$ & 0.00 & 0.00 & 5.00 & 0.00 & 0.00 & 2.70 & 0.00 & 0.00 & 0.00 & 0.00 \\
\hline Diameter & $\mathrm{BC} 1$ & $\mathrm{BC} 2$ & $\mathrm{BC} 3$ & $\mathrm{BC} 4$ & $\mathrm{BC} 5$ & $\mathrm{BC} 6$ & $\mathrm{BC} 7$ & $\mathrm{BC} 8$ & $\mathrm{BC} 9$ & $\mathrm{BC} 10$ \\
\hline $0-0.1$ & 0.00 & 0.00 & 8.33 & 0.00 & 0.00 & 0.00 & 2.33 & 0.00 & 0.00 & 0.00 \\
\hline $0.1-0.2$ & 13.16 & 5.56 & 33.33 & 3.33 & 5.41 & 2.44 & 13.95 & 7.89 & 10.53 & 8.57 \\
\hline $0.2-0.3$ & 13.16 & 5.56 & 30.56 & 16.66 & 8.11 & 21.95 & 20.93 & 13.16 & 7.89 & 8.57 \\
\hline $0.3-0.4$ & 15.79 & 27.76 & 25.00 & 6.67 & 18.92 & 19.51 & 11.63 & 15.79 & 31.58 & 17.14 \\
\hline $0.4-0.5$ & 21.05 & 16.67 & 0.00 & 20.00 & 18.92 & 29.26 & 27.91 & 21.07 & 15.79 & 25.71 \\
\hline $0.5-0.6$ & 15.79 & 25.00 & 2.78 & 20.00 & 18.92 & 12.20 & 4.65 & 23.68 & 21.06 & 11.43 \\
\hline $0.6-0.7$ & 15.79 & 5.56 & 0.00 & 26.67 & 10.80 & 4.88 & 6.97 & 7.89 & 7.89 & 14.29 \\
\hline $0.7-0.8$ & 5.26 & 13.89 & 0.00 & 6.67 & 16.22 & 4.88 & 9.30 & 5.26 & 5.26 & 14.29 \\
\hline $0.8-0.9$ & 0.00 & 0.00 & 0.00 & 0.00 & 0.00 & 2.44 & 2.33 & 5.26 & 0.00 & 0.00 \\
\hline $0.9-1.0$ & 0.00 & 0.00 & 0.00 & 0.00 & 2.70 & 2.44 & 0.00 & 0.00 & 0.00 & 0.00 \\
\hline
\end{tabular}

60 


\section{Supporting Information}

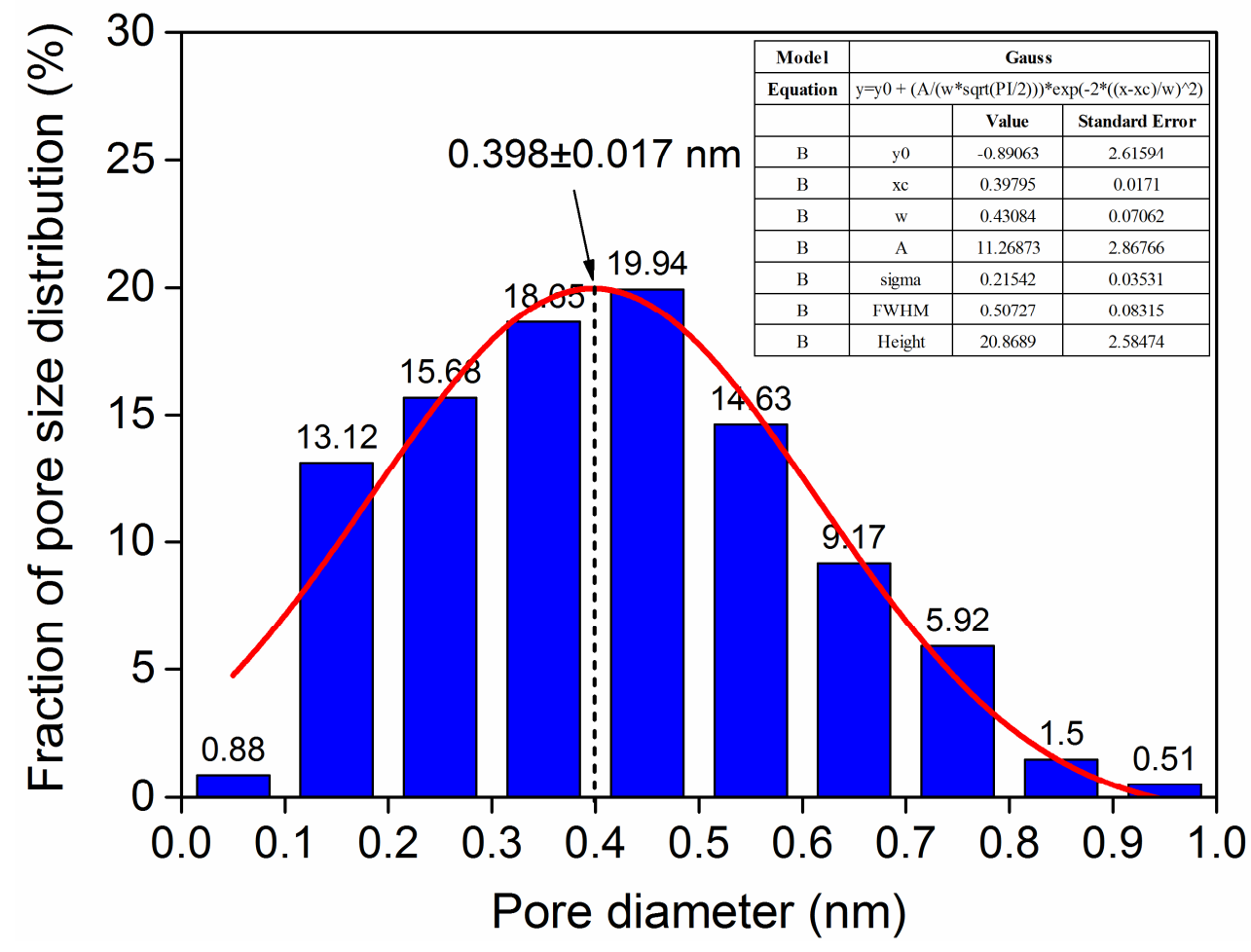

63 Figure S2. Pore size distribution of simulated coal model. 


\section{Supporting Information}

\section{Micro-porosity of coal}

66 There are two distinct phases in the configuration of coal as a kind of porous material, a solid

67 phase known as occupied volume which is occupied by aromatic skeleton, and a void phase

68 known as free volume (pore volume). According to references, the theory and algorithm of

69 Connolly surface were used to calculate the molecular surface and volume of coals. ${ }^{59}$

70 First, the van der Waals (vdW) surface that intersects with the vdW radii of the atoms in the

71 structure is defined. Imagine a spherical "shield" surrounding the atom, and the vdW radius is

72 the closest distance between two non-bonded atoms when their respective shields touch. This

73 envelope is a closed surface and the volume it contained is called the vdW volume $\left(V_{v d W}\right)$. Then,

74 a Connolly surface is at the boundary between the Connolly probe and the atoms (as represented

75 by their scaled vdW radii). This envelope is a closed surface and the volume it contained is

76 called the molecular volume $\left(V_{T}\right)$. The molecular volume is made up of the occupied volume

$77\left(V_{O}\right)$ and free volume $\left(V_{F}\right)$. The occupied and free volume are depicted in Figure 1(b) as gray

78 zone and blue zone, respectively.

79 The occupied volume of the polymers is expressed as 1.3 times the vdW volume of the

80 polymers, and the free volume is the outlines of the vdW surface of polymers. ${ }^{58}$ Therefore, the

81 micro-porosity can be defined as

$82 \quad$ Micro-porosity $=\frac{V_{F}}{V_{T}}=\frac{V_{T}-V_{O}}{V_{T}}=\frac{V_{T}-1.3 \cdot V_{v d W}}{V_{T}}$

83 In this work, there are five atoms existing in our coal molecules $\left(\mathrm{C}_{960} \mathrm{H}_{830} \mathrm{O}_{95} \mathrm{~N}_{20} \mathrm{~S}_{45}\right)$, the vdW

84 radii of $\mathrm{C}, \mathrm{H}, \mathrm{O}, \mathrm{N}$ and $\mathrm{S}$ are $0.170 \mathrm{~nm}, 0.120 \mathrm{~nm}, 0.152 \mathrm{~nm}, 0.155 \mathrm{~nm}$ and $0.180 \mathrm{~nm}$,

85 respectively. Simulation cell of coal with pores is displayed in Figure 1(b). The calculated vdW

86 volume was $13291.38 \AA^{3}$, and the molecular volume was $20736.8 \AA^{3}$. Therefore, the micro-

87 porosity was calculated to be $16.68 \%$. 


\section{Supporting Information}

\section{Formula of three distinct diffusion coefficients}

89 The self-diffusion coefficient $D^{s}\left(\mathrm{~m}^{2} / \mathrm{s}\right)$ covers the average mean-square displacement (MSD)

90 of individual molecules over time. It can also be calculated based on Einstein diffusion law

$91 \quad D^{s}=\frac{1}{6 N} \lim _{t \rightarrow \infty}-\left\langle\sum_{i=1}^{N}\left[\vec{r}_{i}(t)-\vec{r}_{i}(0)\right]^{2}\right\rangle$

92 Where $N$ is the number of diffusion molecules, $\vec{r}_{i}(t)$ is the displacement vector of the $i$-th

93 molecule at time $t$, and the angular bracket $<>$ denotes that the quantity is an ensemble average

94 property.

95 The transport diffusion coefficient is a measure of the flux of a component if a concentration

96 gradient is imposed on a system. The Fick's first law illustrates that the diffusion flux $\boldsymbol{J}$ of

97 particles (or molecular species) is induced by a gradient of concentration $c$ (molecules per unit

98 volume). The extension of Fick's transport equation from the pure component to

99 multicomponent is

$100 \quad(\mathbf{J})=-\left[\mathbf{D}^{\mathbf{t}}\right] \cdot \nabla c$

$101 \quad$ For a binary mixture, Eq.(S3) reads

$102\left(\begin{array}{l}J_{1} \\ J_{2}\end{array}\right)=-\left(\begin{array}{ll}D_{11}^{t} & D_{12}^{t} \\ D_{21}^{t} & D_{22}^{t}\end{array}\right) \cdot\left(\begin{array}{l}\Delta c_{1} \\ \Delta c_{2}\end{array}\right)$

103 From a point view of thermodynamics, the driving force for the diffusion is the gradient in the

104 chemical potential $\mu(\mathrm{J} / \mathrm{mol}),{ }^{37}$ so the Fick's first law can be expressed as

$105 \quad(\mathbf{J})=-[\mathbf{L}] \cdot \nabla \mu$

106 For a binary mixture, Eq.(S5) reads 


\section{Supporting Information}

$107 \quad\left(\begin{array}{l}J_{1} \\ J_{2}\end{array}\right)=-\left(\begin{array}{ll}L_{11} & L_{12} \\ L_{21} & L_{22}\end{array}\right) \cdot\left(\begin{array}{l}\Delta \mu_{1} \\ \Delta \mu_{2}\end{array}\right)$

108 Where $[\mathbf{L}]$ is the symmetric matrix of the Onsager transport coefficients, it can be expressed in

109 the equivalent Einstein form. ${ }^{52}$ For a mixture of species $i$ and $j$

110

$$
L_{i j}=\frac{1}{6 V k_{B} T} \lim _{t \rightarrow \infty} \frac{1}{t}\left\langle\sum_{m=1}^{N_{i}}\left[\vec{r}_{m i}(t)-\vec{r}_{m i}(0)\right] \cdot \sum_{n=1}^{N_{j}}\left[\vec{r}_{n j}(t)-\vec{r}_{n j}(0)\right]\right\rangle \quad i, j=1,2
$$

111 Where $k_{B}$ is the Boltzmann constant $\left(1.38 \times 10^{-23} \mathrm{~J} \cdot \mathrm{K}^{-1}\right), V$ is the volume $\left(\mathrm{m}^{3}\right), T$ is the temperature

$112(\mathrm{~K}), \vec{v}_{m i}(t)$ is the velocity vector of the $m$-th molecule of species $i$ at time $t$, and $N_{i}$ is the total

113 number of molecules of species $i$.

114 Replacing the chemical potential in Eq.(S6) with the fugacity $f$, we can get

$115\left(\begin{array}{l}J_{1} \\ J_{2}\end{array}\right)=-k_{B} T \cdot\left(\begin{array}{ll}L_{11} & L_{12} \\ L_{21} & L_{22}\end{array}\right)\left(\begin{array}{cc}1 / c_{1} & 0 \\ 0 & 1 / c_{2}\end{array}\right)\left(\begin{array}{ll}\Gamma_{11} & \Gamma_{12} \\ \Gamma_{21} & \Gamma_{22}\end{array}\right) \cdot\left(\begin{array}{l}\Delta c_{1} \\ \Delta c_{2}\end{array}\right)$

116 Where $\Gamma_{i j}$ is the matrix of the thermodynamic factor. For a binary mixture, it can be defined as

$117 \quad \Gamma_{i j}=\frac{c_{i}}{c_{j}} \frac{\partial \ln f_{i}}{\partial \ln c_{j}} \quad i, j=1,2$

118 According to Eq.(S4), (S8), and (S9), the transport diffusion coefficients for binary mixture

119 can be expressed as 


\section{Supporting Information}

120

$$
\left[\mathbf{D}^{\mathbf{t}}\right]=\left(\begin{array}{ll}
D_{11}^{t} & D_{12}^{t} \\
D_{21}^{t} & D_{22}^{t}
\end{array}\right)=k_{B} T \cdot\left(\begin{array}{ll}
L_{11} & L_{12} \\
L_{21} & L_{22}
\end{array}\right)\left(\begin{array}{cc}
1 / c_{1} & 0 \\
0 & 1 / c_{2}
\end{array}\right)\left(\begin{array}{ll}
\Gamma_{11} & \Gamma_{12} \\
\Gamma_{21} & \Gamma_{22}
\end{array}\right)
$$

121

$$
\left\{\begin{array}{l}
D_{i i}^{t}=\frac{k_{B} T}{c_{i}}\left(L_{i i} \cdot \frac{\partial \ln f_{i}}{\partial \ln c_{i}}+L_{i j} \cdot \frac{\partial \ln f_{j}}{\partial \ln c_{i}}\right) \\
D_{i j}^{t}=\frac{k_{B} T}{c_{j}}\left(L_{i i} \cdot \frac{\partial \ln f_{i}}{\partial \ln c_{j}}+L_{i j} \cdot \frac{\partial \ln f_{j}}{\partial \ln c_{j}}\right)
\end{array} i, j=1,2 ; i \neq j\right.
$$

122 In Eq.(S10), the product of the first three items on the right side of equation can be considered

123 as so-called corrected diffusion coefficient $D^{c}\left(\mathrm{~m}^{2} / \mathrm{s}\right)$. That is, the transport diffusion coefficient

124 can be separated into a corrected diffusion part and a thermodynamic part: ${ }^{37}$

$$
\left[\mathbf{D}^{\mathbf{t}}\right]=\left[\mathbf{D}^{\mathbf{c}}\right] \cdot[\boldsymbol{\Gamma}]=\left(\begin{array}{ll}
D_{11}^{c} & D_{12}^{c} \\
D_{21}^{c} & D_{22}^{c}
\end{array}\right)\left(\begin{array}{ll}
\Gamma_{11} & \Gamma_{12} \\
\Gamma_{21} & \Gamma_{22}
\end{array}\right)
$$

126 All three diffusion coefficients are functions of the adsorbed species concentration, and they 127 are only equal in the limit $c \rightarrow 0$. 


\section{Supporting Information}

\section{Initial conformation for MD simulation}

130 The size of adsorption conformation obtained by GCMC simulation was $27.5 \times 27.5 \times 27.5 \AA^{3}$.

131 In the simulation box, the numbers of species are too little $-\mathrm{CO}_{2}$ (about 16-20 molecules/unit

132 cell), $\mathrm{CH}_{4}$ (only about 2-4 molecules/unit cell). According to Smit and Maesen, the diffusion

133 coefficient is related to density fluctuations in a system with periodic boundary condition, ${ }^{37}$ so

134 the system must be sufficiently large to compute a diffusion coefficient accurately. Therefore, if

135 the simulation box $\left(27.5 \times 27.5 \times 27.5 \AA^{3}\right)$ is used as the initial conformation for MD simulation,

136 the calculated diffusion coefficients may be less accurate, especially for $\mathrm{CH}_{4}$. Sanborn and Snurr

137 have studied the diffusion coefficient of binary mixtures of $\mathrm{CF}_{4}$ and $\mathrm{n}$-alkanes in faujasite, the

138 simulation box is $2 \times 2 \times 2$ unit cells, and the edge length of each unit cell is $24.258 \AA^{3} .^{52}$

139 In our study, the total molecules of our unit cell are much more than Sanborn and Snurr ${ }^{52}$, if

140 we choose the same simulation box $(2 \times 2 \times 2$ unit cells $)$, the CPU-time required for the calculation

141 of diffusion coefficient is tremendous. Therefore, the simulation box is only enlarged fourfold

$142(2 \times 2 \times 1)$, which means that the simulation box is $55 \times 55 \times 27.5 \AA^{3}$. An example of the molecular

143 configuration sampled for species diffusion in the bituminous coal model is shown in Figure S3. 


\section{Supporting Information}

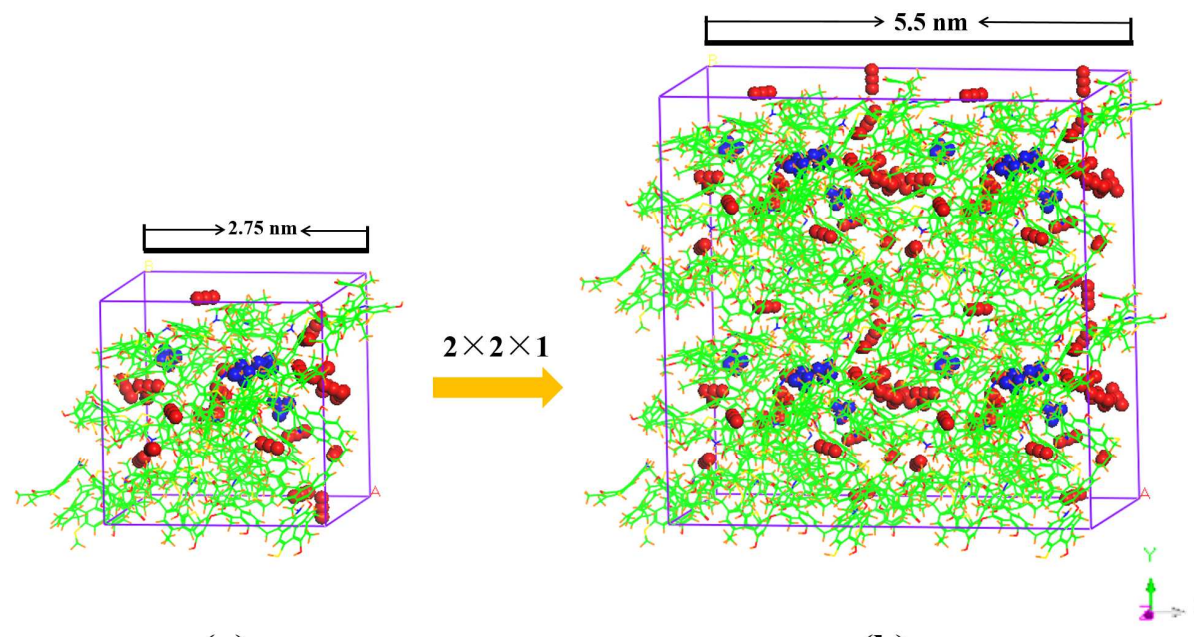

144

(a)

(b)

145 Figure S3. Example of the molecular configuration sampled for diffusion of the $\mathrm{CO}_{2}-\mathrm{CH}_{4}$

146 mixture in the bituminous coal model. (a) Adsorption conformation obtained by GCMC

147 simulation. (b) Initial conformation for $\mathrm{MD}$ simulation. $\mathrm{CO}_{2}$ and $\mathrm{CH}_{4}$ molecules are depicted in 148 red and blue color, respectively. 


\section{Supporting Information} absolute adsorption of $\mathrm{CO}_{2}-\mathrm{CH}_{4}$ mixture on coals, and results are depicted in Figure $\mathbf{S 4}$ and

Figure S5. It is seen that the absolute adsorption amount of each species at a given fugacity

154 decreases with rising temperature. This is ascribed to the exothermic nature of thermodynamical 155 adsorption process.

156 For the description of adsorption, because the chemical potentials of equilibrium phases are 157 proportional to the logarithm of fugacity, the absolute amount may be considered as a function of 158 gas-phase fugacity. ${ }^{55}$ Here, we used the Langmuir equation to describe the absolute adsorption of 159 binary mixture $\left(\mathrm{CO}_{2}\right.$ and $\left.\mathrm{CH}_{4}\right)$ on coals, which is suggested by the fact that the fluid-solid 160 interaction is greater than the fluid-fluid interaction under supercritical conditions. For this 161 model, the absolute amount adsorbed is given by

$162 \quad n_{\text {absolute }}=\frac{n_{\max } \cdot b \cdot f}{1+b \cdot f}$

163 where $n_{\max }$ is the maximum adsorbed amount $(\mathrm{mmol} / \mathrm{g}) ; f$ is the fugacity of the gas phase

$164(\mathrm{MPa}) ; b$ is the adsorption affinity constant.

165 Here, the Origin 9.0 software was used to analyze the data. To fit the species absolute 166 adsorption isotherms, the LangmuirEXT1 function was also selected. The expression of the 167 LangmuirEXT1 function is in the following:

$168 \quad y=\frac{a \cdot b \cdot x^{1-c}}{1+b \cdot x^{1-c}}$

169 When applying to our model, $y$ is the absolute adsorption amount $(\mathrm{mmol} / \mathrm{g}) ; a=n_{\max }, x=f$, $170 c=0, b$ is the adsorption affinity constant. 


\section{Supporting Information}

171 In Figure S4, the fitting results show that the absolute adsorption isotherms of $\mathrm{CO}_{2}$ and $\mathrm{CH}_{4}$

172 are consistent with the Langmuir model, and the maximum adsorbed amounts of $\mathrm{CO}_{2}$ and $\mathrm{CH}_{4}$

173 are also decrease with rising temperature, while the absolute adsorption isotherms of $\mathrm{CO}_{2}$ are

174 higher than $\mathrm{CH}_{4}$ under the same condition. Moreover, Figure S5 depicts that the absolute

175 adsorption amounts of $\mathrm{CO}_{2}$ or $\mathrm{CH}_{4}$ increase with the rising of its own bulk mole fraction. It

176 should be pointed out that the change of absolute adsorption isotherms for the mixture species is

177 slighter than pure $\mathrm{CO}_{2}$ and pure $\mathrm{CH}_{4}{ }^{54}$ It is indicated that the mixture adsorption in coal

178 ultramicropores is restricted by the interaction between $\mathrm{CO}_{2}$ and $\mathrm{CH}_{4}$.
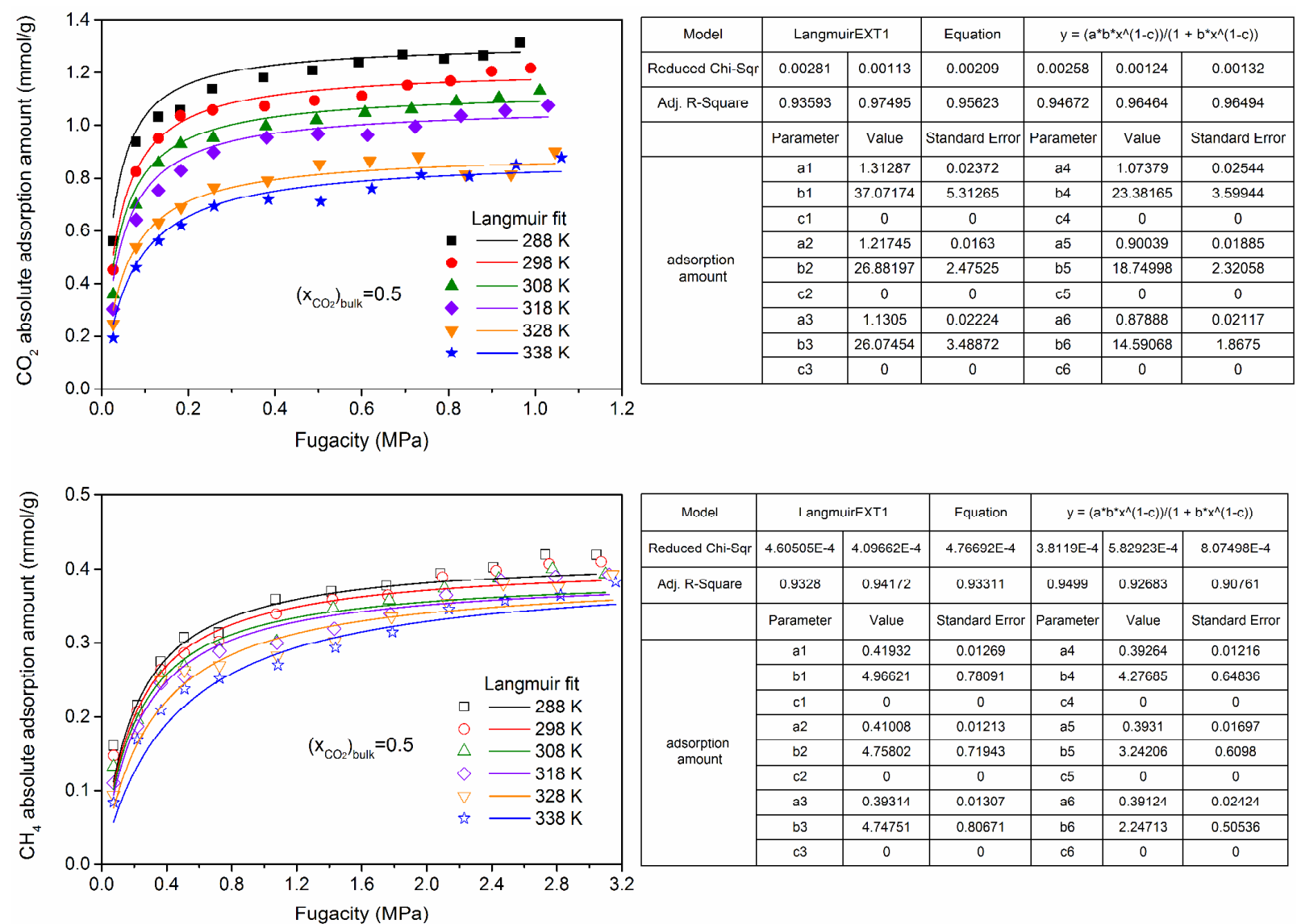

Figure S4. Absolute adsorption isotherms of the $\mathrm{CO}_{2}-\mathrm{CH}_{4}$ mixture in coal ultramicropores at

$181\left(x_{\mathrm{CO}_{2}}\right)_{\text {bulk }}=0.5$, i.e. $\left(x_{\mathrm{CH}_{4}}\right)_{\text {bulk }}=0.5$. 


\section{Supporting Information}

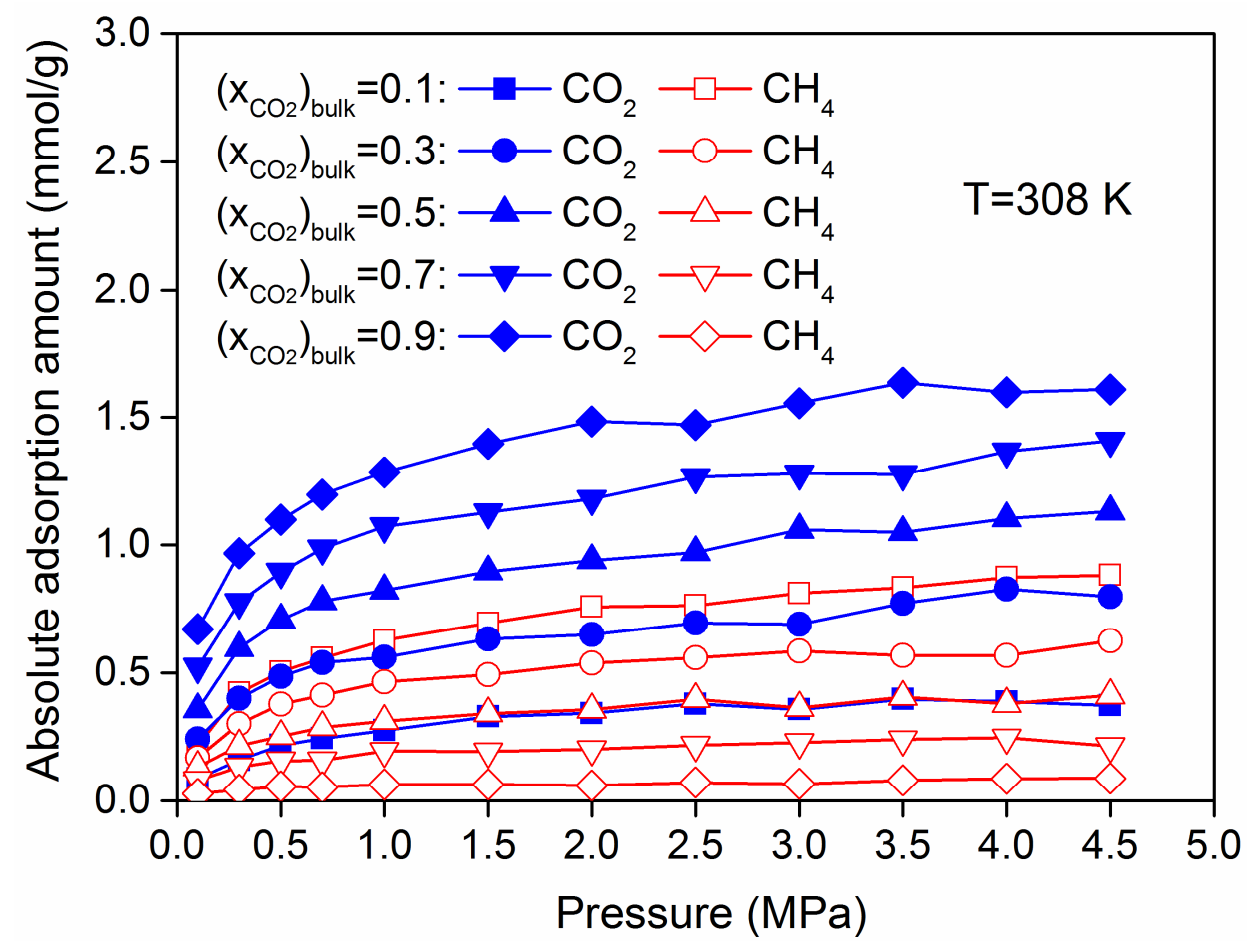

Figure S5. Absolute adsorption isotherms of the $\mathrm{CO}_{2}-\mathrm{CH}_{4}$ mixture in coal ultramicropores at $184308 \mathrm{~K}$. 


\section{Supporting Information}

186

187 In this work, the data of thermodynamic factors obtained by GCMC method are listed in Table

188 S2 and Table S3. The Standard errors were determined from the slopes of the least squares fits.

189

Table S2. Data of thermodynamic factors at $\left(x_{\mathrm{CO}_{2}}\right)_{\text {bulk }}=0.5$.

\begin{tabular}{cccccccc}
\hline terms & Expression & $288 \mathrm{~K}$ & $298 \mathrm{~K}$ & $308 \mathrm{~K}$ & $318 \mathrm{~K}$ & $328 \mathrm{~K}$ & $338 \mathrm{~K}$ \\
\hline$\Gamma_{11}$ & $c_{1} \cdot \partial \ln f_{1} / c_{1} \cdot \partial \ln c_{1}$ & $4.41 \pm 0.29$ & $3.73 \pm 0.22$ & $3.25 \pm 0.20$ & $3.04 \pm 0.20$ & $2.76 \pm 0.15$ & $2.44 \pm 0.15$ \\
$\Gamma_{12}$ & $c_{1} \cdot \partial \ln f_{1} / c_{2} \cdot \partial \ln c_{2}$ & $12.42 \pm 0.78$ & $10.24 \pm 0.60$ & $8.62 \pm 0.60$ & $7.56 \pm 0.47$ & $6.59 \pm 0.52$ & $5.49 \pm 0.37$ \\
$\Gamma_{21}$ & $c_{2} \cdot \partial \ln f_{2} / c_{1} \cdot \partial \ln c_{1}$ & $1.31 \pm 0.09$ & $1.26 \pm 0.08$ & $1.24 \pm 0.08$ & $1.13 \pm 0.08$ & $1.07 \pm 0.06$ & $1.07 \pm 0.07$ \\
$\Gamma_{22}$ & $c_{2} \cdot \partial \ln f_{2} / c_{2} \cdot \partial \ln c_{2}$ & $3.68 \pm 0.24$ & $3.46 \pm 0.21$ & $3.28 \pm 0.24$ & $2.82 \pm 0.18$ & $2.54 \pm 0.21$ & $2.41 \pm 0.17$ \\
\hline
\end{tabular}

190

Table S3. Data of thermodynamic factors at $308 \mathrm{~K}$.

\begin{tabular}{cccccc}
\hline terms & Expression & $\left(x_{\mathrm{CO}_{2}}\right)_{\text {bulk }}=0.1$ & $\left(x_{\mathrm{CO}_{2}}\right)_{\text {bulk }}=0.2$ & $\left(x_{\mathrm{CO}_{2}}\right)_{\text {bulk }}=0.3$ & $\left(x_{\mathrm{CO}_{2}}\right)_{\text {bulk }}=0.4$ \\
\hline$\Gamma_{11}$ & $c_{1} \cdot \partial \ln f_{1} / c_{1} \cdot \partial \ln c_{1}$ & $2.39 \pm 0.17$ & $2.93 \pm 0.17$ & $3.10 \pm 0.17$ & $3.35 \pm 0.22$ \\
$\Gamma_{12}$ & $c_{1} \cdot \partial \ln f_{1} / c_{2} \cdot \partial \ln c_{2}$ & $1.08 \pm 0.07$ & $2.71 \pm 0.19$ & $4.11 \pm 0.30$ & $6.36 \pm 0.39$ \\
$\Gamma_{21}$ & $c_{2} \cdot \partial \ln f_{2} / c_{1} \cdot \partial \ln c_{1}$ & $6.01 \pm 0.45$ & $3.09 \pm 0.18$ & $2.20 \pm 0.13$ & $1.60 \pm 0.11$ \\
$\Gamma_{22}$ & $c_{2} \cdot \partial \ln f_{2} / c_{2} \cdot \partial \ln c_{2}$ & $2.73 \pm 0.19$ & $2.86 \pm 0.21$ & $2.92 \pm 0.23$ & $3.04 \pm 0.20$ \\
\hline terms & $\left(x_{\mathrm{CO}_{2}}\right)_{\text {bulk }}=0.5$ & $\left(x_{\mathrm{CO}_{2}}\right)_{\text {bulk }}=0.6$ & $\left(x_{\mathrm{CO}_{2}}\right)_{\text {bulk }}=0.7$ & $\left(x_{\mathrm{CO}_{2}}\right)_{\text {bulk }}=0.8$ & $\left(x_{\mathrm{CO}_{2}}\right)_{\text {bulk }}=0.9$ \\
\hline$\Gamma 11$ & $3.24 \pm 0.20$ & $3.72 \pm 0.22$ & $3.72 \pm 0.17$ & $3.82 \pm 0.25$ & $4.18 \pm 0.17$ \\
$\Gamma 12$ & $8.62 \pm 0.60$ & $12.37 \pm 1.03$ & $21.42 \pm 1.66$ & $31.68 \pm 1.59$ & $83.84 \pm 5.75$ \\
$\Gamma 21$ & $1.24 \pm 0.08$ & $0.83 \pm 0.05$ & $0.59 \pm 0.03$ & $0.39 \pm 0.03$ & $0.18 \pm 0.01$ \\
$\Gamma 22$ & $3.28 \pm 0.24$ & $2.77 \pm 0.23$ & $3.42 \pm 0.28$ & $3.27 \pm 0.16$ & $3.59 \pm 0.25$ \\
\hline
\end{tabular}

191

192 


\section{Supporting Information}

\section{Pressure dependence on diffusion coefficients}

194 The pressure dependence of diffusion coefficients for the $\mathrm{CO}_{2}-\mathrm{CH}_{4}$ mixture in coal

195 ultramicropores was investigated at $298 \mathrm{~K}$, bulk $\mathrm{CO}_{2}$ mole fractions $\left(x_{\mathrm{CO}_{2}}\right)_{\text {bulk }}=0.5$ and the result

196 is displayed in Figure S6. It is seen that the matrices of the transport diffusion coefficients first

197 increase to reach a peak and then fall with the pressure rising, but the tendency is ambiguous for 198 the self- and matrices of the corrected diffusion coefficients. For the $\mathrm{CO}_{2}-\mathrm{CH}_{4}$ mixture, the 199 transport mechanisms are the same as pure $\mathrm{CO}_{2}$ and pure $\mathrm{CH}_{4}$ in coal ultramicropores. With the 200 pressure rising, dominating mechanism for gas diffusion in coal ultramicropores changes from 201 surface diffusion to configurational diffusion, which can be addressed by the diffusion activation

202 energy and coal swelling, as described in our previous study. ${ }^{54}$ Moreover, it is also illustrated 203 that the off-diagonal terms of transport diffusion coefficient matrix are important for the 204 preferential diffusive species, i.e. $\mathrm{CO}_{2}$. 


\section{Supporting Information}
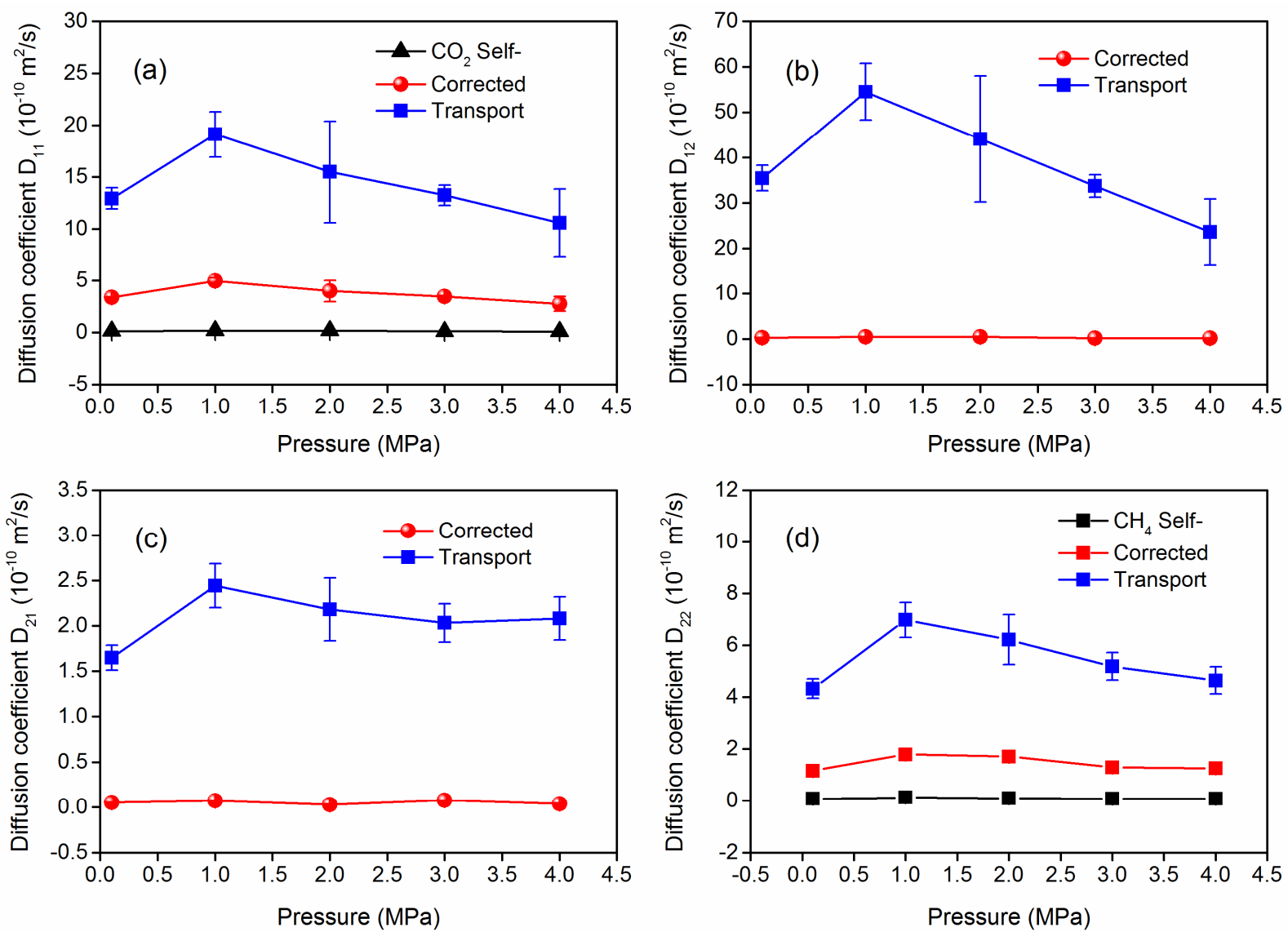

206 Figure S6. Diffusion coefficients of the $\mathrm{CO}_{2}-\mathrm{CH}_{4}$ mixture in coal ultramicropores versus

207 Pressure at $298 \mathrm{~K}$ and $\left(x_{\mathrm{CO}_{2}}\right)_{\text {bulk }}=0.5$. The subscript ' 1 ' refers to $\mathrm{CO}_{2}$, and '2' refers to $\mathrm{CH}_{4}$. The 208 error bars were obtained from the slopes of the least squares fits. 